\title{
Fast and Slow Ion Diffusion Processes in Lithium Ion Pouch Cells during Cycling Observed with Fiber Optic Strain Sensors
}

Lars Wilko Sommer ${ }^{1}$, Peter Kiesel ${ }^{1}$, Anurag Ganguli ${ }^{1}$, Alexander Lochbaum ${ }^{1}$, Bhaskar Saha ${ }^{1}$, Julian Schwartz ${ }^{1}$, Chang-Jun Bae ${ }^{1}$, Mohamed Alamgir ${ }^{2}$, and Ajay Raghavan ${ }^{1 *}$

${ }^{1}$ Palo Alto Research Center (PARC, a Xerox Company), Palo Alto, CA 94304, U.S.A.

${ }^{2}$ LG Chem Power, Troy, MI 48083, U.S.A.

\begin{abstract}
Cell monitoring for safe capacity utilization while maximizing pack life and performance is a key requirement for effective battery management and encouraging their adoption for cleanenergy technologies. A key cell failure mode is the build-up of residual electrode strain over time, which affects both cell performance and life. Our team has been exploring the use of fiber optic (FO) sensors as a new alternative for cell state monitoring. In this present study, various charge-cycling experiments were performed on Lithium-ion pouch cells with a particular class of FO sensors, fiber Bragg gratings (FBGs), that were externally attached to the cells. An overshooting of the volume change at high SOC that recovers during rest can be observed. This phenomenon originates from the interplay between a fast and a slow Li ion diffusion process, which leads to non-homogeneous intercalation of $\mathrm{Li}$ ions. This paper focuses on the strain relaxation processes that occur after switching from charge to no-load phases. The correlation of the excess volume and subsequent relaxation to SOC as well as temperature is discussed. The
\end{abstract}

\footnotetext{
* Corresponding author. Email: raghavan@parc.com; Phone: 1-650-812-4724; Fax: 1-650-812-4334
} 
implications of being able to monitor this phenomenon to control battery utilization for long life are also discussed.

\section{Introduction}

In recent years, the market for alternative drive technologies such as electric vehicles (EVs) and hybrid electric vehicles (HEVs) has experienced considerable growth. However, the widespread adoption of these green mobility options is still limited by factors such as high costs, battery lifetime, driving range anxiety and the susceptibility to unexpected failures. Effective control and management of cell charge and discharge by battery management systems (BMS) is essential for good performance [1]-[3]. Current BMS rely on monitoring conventional external parameters such as voltage, current and external temperature, typically with one measurement made per group of 2-10 cells, to estimate state of charge (SOC) and state of health ( $\mathrm{SOH}$ ). The use of these weakly informative parameters limits the estimation accuracy of SOC and SOH and contributes to over-conservative usage and over-engineering of a battery pack [2]. Using additional informative cell parameters such as internal temperature or electrode volume change due to lithium intercalation has the potential to improve SOC/SOH estimation accuracy, which in turn can lead to optimal battery-pack design and capacity utilization. However, available options to sense these internal parameters are limited by the corrosive and electrically noisy environment of batteries and demanding constraints on potential sensors such as cost, size, non-invasiveness, and robustness. Fiber optic (FO) sensors exhibit a small form-factor, immunity to electromagnetic interference and electrostatic discharge, and multiplexing capabilities. These attributes make them attractive candidates for reliable state monitoring in battery packs $[4,5]$ 
In this study, a particular class of FO sensors, fiber Bragg grating (FBG) sensors sensitive to temperature and strain were externally attached to lithium ion pouch cells. The potential of FBG sensors as a promising solution for monitoring cell parameters such as strain and temperature was already discussed in our previous work [6]. Multiple charge and discharge cycles demonstrated the excellent repeatability of the FBG strain signal and indicated consistent features in the strain signal that provide additional information about the cell state. FBGs can also be used for directly monitoring electrode strain and temperature inside the cell; results from such internal configurations accomplished by our team will be reported elsewhere in the future. In the present work, a more detailed understanding of the external FO sensing signal in relation to the underlying electrochemical processes is explored.

The functional principle of lithium-ion batteries is based on lithium intercalation. The insertion/release of lithium ions into/from the electrode active materials is associated with structural changes of the electrode active materials and consequently with volume change. In general, the volume change is predominantly caused by the anode material. Thus, the battery expands during charge and contracts during discharge [7]-[9]. Previous experiments indicated an overshooting of the volume change (also referred to as "excess volume change" within this paper) at the end of charge and volume relaxation in the subsequent no load phase. The impact of these overshoots has to be elucidated with regard to the utility of the strain signal as input parameter for BMS.

Similar characteristics at the end of charge of lithium ion batteries are reported by Lee et al. [8]. They noted a thickness change that was heavily increasing at the end of the constant current $(\mathrm{CC})$ charge phase. The increase diminished during the constant voltage $(\mathrm{CV})$ charging phase and the thickness started to relax. They explained this behavior with a non-uniform 
intercalation/de-intercalation at high SOC. Wang et al [10]- [11] reported a similar excess volume change at the end of charge and a volume relaxation in the subsequent rest phase. They attributed these effects to the slow diffusion of lithium ions in the active material and the slow structural change in the outer region of the electrode particle. As observed from extended cycling experiments in [10]- [11], this excess volume change can lead to residual strain build up over time, affecting cell performance and life. Therefore, monitoring and controlling this phenomenon can be very useful to maximize cell life and performance in cutting-edge applications such as $\mathrm{xEVs}$ and satellite batteries since electrode volume change is identified as a key mechanism for capacity fading [10]-[11].

In this work, various charge experiments were performed to investigate a correlation of the overshooting volume change and the amount of intercalated lithium ions. In particular, this paper focuses on the strain relaxation processes that occur after switching from charge to no-load phases. The relaxation phenomena was measured at various temperatures and approximated by a decaying exponential function to explore the temperature dependence of the underlying process.

\section{Experimental setup}

Commercial LG Chem lithium-ion pouch cells are used for our experiments. The cathode is a blended material composed of spinel/NMC (nickel manganese cobalt oxide) while the anode material is a blended material with graphite. The nominal capacity of the cells is $15 \mathrm{Ah}$.

Two FBG sensors are used to monitor cell state in our experimental set-up as shown in Figure 1. FBG sensors exhibit a periodic modulation of the refractive index along the core of the fiber. This modulation is inscribed into silica fiber and acts as a Bragg mirror reflecting a certain wavelength, known as the Bragg wavelength, of the incoming light. The fibers are coated with 
polyimide, and the core and cladding diameters are $9 \mu \mathrm{m}$ and $125 \mu \mathrm{m}$, respectively. The Bragg wavelengths for the two FBGs are $1555 \mathrm{~nm}$ and $1560 \mathrm{~nm}$. While for this initial study two separate optical fibers with FBGs are used, in principle, for deployed architectures the two FBGs can be multiplexed on the same optical fiber.

As cells undergo charging and discharging, the electrodes' volumes experience changes, which manifest as strain on the cell surface. In addition, the cell temperature also changes due to thermic electrochemical reactions and resistive heating. Two FBG sensors are used in order to measure both the temperature changes as well as cell strain due to electrode volume change. The first FBG is bonded at two points to the surface of the pouch cell with epoxy. The bonding lengths are approximately $1 \mathrm{~cm}$. This FBG responds to both strain and temperature changes. The strain is caused due to electrode volume change, as well as thermal expansion or contraction. The second FBG, also referred to as the reference FBG sensor, is loosely attached to the cell skin with a heat conducting paste. As such, it is sensitive only to temperature changes. The wavelength shift due to the strain from the electrode volume change alone is recovered by subtracting the wavelength shift observed with the bonded FBG multiplied by a suitable constant from the wavelength shift observed by the reference FBG. The constant factor is determined empirically by conducting a calibration experiment in an environmental chamber where the cell is heated to various temperatures but not electrically cycled.

The reflected wavelength peaks of the FBG sensors are monitored by a commercial readout, the National Instruments ${ }^{\mathrm{TM}}$ PXIe-4844 optical sensor interrogator (OSI). The accuracy of the measured peak wavelength of the FBG sensors is $\sim 1 \mathrm{pm}$. A programmable battery analyzer is used to run defined charge and discharge cycles. The standard cycle employed here consisted of charging at a constant current of $7.5 \mathrm{~A}$ to a cutoff voltage of $4.15 \mathrm{~V}$, charging at a CV of 4.15 
$\mathrm{V}$ to a cutoff current of $750 \mathrm{~mA}$ and discharging at a constant current of $7.5 \mathrm{~A}$ to a cutoff voltage of $3.0 \mathrm{~V}$. The rest time between charge and discharge was set to $2 \mathrm{~h}$.

\section{Results and discussions}

Figure 2 shows the wavelength shift measured with the bonded FBG sensor, the loosely attached reference FBG sensor as well as the temperature compensated strain signal as a function of time for a standard cycle. The standard cycle is divided into five regions, as illustrated in Figure 2. Standard charge and standard discharge are labeled as II and IV, respectively. The initial rest period and the rest periods after charge and discharge are labeled as I, III, and V. The corresponding cell voltage and current are given in the upper subplot. The reflected wavelength of both sensors was zeroed at the beginning of the charge cycle. Note that a wavelength shift of 1 pm corresponds to a strain of approximately $1 \mu \varepsilon$ in the extracted strain signal (or a temperature change of approximately $0.1^{\circ} \mathrm{C}$ in the loose $\mathrm{FBG}$ signal).

As mentioned earlier, the reference sensor is sensitive only to temperature variations at the cell surface. As expected, the corresponding measured wavelength shift and consequently the temperature are mainly increasing during charge and discharge. The increase in temperature is caused by an increasing internal resistance such as the polarization resistance and the associated Joule heating. However, there are also distinct SOC values during charge and discharge where endothermic reactions can be observed.

As shown in Figure 2, the wavelength shift of the reference sensor is decreasing after the transition from charge as well as discharge to no load and converges to the initial values of wavelength shift due to temperature and strain relaxation. The temperature decrease in region I is due to the temperature relaxation after the previous discharge cycle. A more detailed discussion 
of the temperature signal from the reference FBG sensor signal as well as a comparison to a conventional thermistor signal is offered in our team's previously published work [6]. As mentioned earlier, this present paper will mainly focus on the strain relaxation processes that occur after switching from charge to no-load phases.

The wavelength shift of the bonded FBG sensor is a superposition of mechanical strain and temperature effects (in the form of thermal strain). For example, the sensitivity to temperature variations is clearly visible by the strong increase in the wavelength shift signal at the end of discharge. Therefore the wavelength shift of the bonded FBG sensor needs to be temperature compensated as described above. In the following, the temperature compensated strain signal is referred to as the extracted strain signal.

In reality, this temperature compensation is imperfect as it relies on the external skin temperature monitored by the loosely attached FBG. In practice, at higher $\mathrm{C}$ rates and/or for thicker cells, there can be a significant temperature gradient between internal core temperature and external skin temperature [12]. However, in the present study, since thinner pouch cells are used at low $\mathrm{C}$ rates for the initial experiments, these effects are not expected to be significant.

As has been reported in the literature (e.g., [13]), the SOC distribution across the cell, particularly for larger-format cells, can be inhomogeneous, and increasingly so as the cell ages. Correspondingly, some differences in local SOC can be expected over the electrode surface. However, as our paper reports on external sensing cell skin strain results, the measured signals are, in a sense, an average of the changes across the layers.

Furthermore, intuitively strain at a point in the electrode is not only affected by local SOC but also by expansion/contraction from lithiation/delithiation at other points in the electrode layer (since they are all mechanically part of the same electrode structure). Therefore, the strain 
measured at one point is expected to be indicative of SOC over a larger area rather than just the local SOC at that point. All the same, it would be interesting to explore the spatial spread in strain measurements over the cell surface in future studies.

In the initial no-load phase (region I in Fig. 2), the wavelength shift of the temperature compensated strain signal (also referred to as the "extracted" strain signal here), is almost constant. In region II the strain signal is increasing during constant current charge. Towards the end of the charge phase the strain signal shows further increase and reaches its maximum value in the CV charging phase. In the following rest period (region III) the strain signal starts decreasing, indicating a relaxation process. At the end of the rest period a residual wavelength shift and consequently a residual strain is observable. During the subsequent discharge cycle the strain converges towards the initial value. The volume expansion during charge and the contraction during discharge are associated with the lithium ion insertion/release and the corresponding lattice expansion and contraction of the electrode active material. In general, the volume change is dominated by the active material of the anode [8]: the volume change expected in the anode for this cell is approximately $10 \%$ over a full charge cycle while it is just $3 \%$ in the cathode over a full discharge cycle.

The cell volume change over charging/discharging is not expected to be isotropic: earlier work [7] has suggested that is predominantly driven by the electrode thickness change with intercalation. However, some resultant electrode lateral expansion due to the Poisson's effect is expected (which may explain why strong cell skin lateral expansion signals are observed by us here). The cell volume change is intuitively expected to be related to the measured lateral strain signal by a 3rd-degree polynomial function. The specific function will also be influenced by the expansion coefficient of the cell skin and the strain transfer coefficient between cell skin and the 
fiber, which is affected by the fiber coating and the bonding material. This hypothesis needs to be confirmed experimentally in future studies.

In addition to the residual strain after charging to a SOC level of $100 \%$, distinctive features during charge and discharge as well as a relaxation phenomenon after charging are indicated. These features in the strain curve, which can be associated to the different intercalation stages of $\mathrm{Li}$ ions in the anode material, will be discussed in detail in another paper to be published elsewhere. The relaxation phenomenon after charging is examined in more detail next here. Various experiments are performed under various cycling and external temperature conditions.

As already mentioned, the strain signal exhibits an overshoot towards the end of the charge cycle and reaches its maximum in the CV phase. Subsequently the strain starts decreasing. At the transition of the $\mathrm{CV}$ charge and no load phase the rate of strain decrease becomes steeper and exhibits a curve typical of a relaxation process. The temperature spike at the end of charge due to internal Ohmic dissipation is excluded in the extracted strain signal as the temperature relaxation is compensated by the reference FBG sensor. This hypothesis gets confirmed by the observation that the rest period after discharge indicates no pronounced relaxation in the extracted strain signal, even though the temperature value at the end of discharge is much higher compared to that at the end of charge.

Initially, the relation between strain relaxation and SOC, (total amount of intercalated/deintercalated lithium) is examined. For this purpose, one cell was stepwise charged with $\mathrm{C} / 2$ (charging with constant current which would allow a full charge in 2 hours) to various SOC levels. Based on the nominal capacity of 15 Ah the respective cut-off criteria for SOC levels of $10 \%, 30 \%, 50 \%$ and $80 \%$ were set to $1.5 \mathrm{Ah}, 4.5 \mathrm{Ah}, 7.5 \mathrm{Ah}$ and $12 \mathrm{Ah}$ total amount of charged 
capacity. The cut-off criterion for SOC of $100 \%$ was similar to the cut-off criteria of the standard cycles specified above. At each SOC level the rest time was set to 2 hours. The corresponding wavelength shifts of the bonded FBG sensor and the reference FBG sensor as well as the extracted strain signal are shown in Figure 3 (a). Each charge step is labeled with C whereas the subsequent rest periods indicate the corresponding SOC.

The wavelength shift of the reference FBG illustrates the temperature changes during each charge step. In the subsequent rest phases the temperature is relaxing to the initial temperature. Over the first step up to SOC level of $10 \%$, the temperature decreases with increasing SOC due to an endothermic reaction. For all other steps, the temperature increases with increasing SOC.

The extracted strain signal shows a continuously increasing wavelength shift during charging for each step except for the last step corresponding to charging to SOC level of $100 \%$. The non-monotonic behavior of the wavelength shift during the CV charging phase leading up to the SOC level of $100 \%$ has been discussed earlier in this paper. In the rest phases following charging, the strain signals exhibit varying characteristics for the different SOCs. At low SOCs no relaxation is distinguishable whereas at high SOCs a clear relaxation process is observable. To highlight these variations, the extracted wavelength shifts during the rest phases in particular at each SOC level are further compared in Figure 3 (b).

The increase in extracted strain signal with higher SOC is consistent with the expected electrode phenomenon of volume expansion due to lithium intercalation. However, the strain increase is nonlinear with increasing SOC: in particular, the extracted strain signal doubles in value going from SOC level of $80 \%$ to $100 \%$. The strain signal exhibits no distinctive relaxation at SOC levels of $10 \%, 30 \%$ and $50 \%$ whereas at $80 \%$ and in particular at $100 \%$ a clear relaxation 
is observable. The strain signal relaxes by $\sim 15 \%$ (from $85 \mathrm{pm}$ to $75 \mathrm{pm}$ ) at SOC level of $80 \%$ and by $\sim 30 \%$ (185pm to $145 \mathrm{pm})$ at SOC level of $100 \%$. These experiments indicate that the pouch cells experience an excess volume change toward the end of charge (at high SOCs), which relaxes within a few tens of minutes.

As described above, the volume change of lithium ion batteries is caused by lithium intercalation/de-intercalation into the electrode active materials. During charging, lithium ions get extracted from the cathode material and intercalate into the graphite anode; lithium ions are inserted in the outer region of the graphite particle at the electrode/electrolyte interphase resulting in a higher lithium ion concentration compared to the inner electrode region. Consequently, the concentration gradient in the graphite particle is the driving force for lithium diffusion. However, the lithium ion diffusion into the inner electrode regions is relatively slow compared to the lithium intercalation due to the somewhat higher C-rate used here in these experiments. Thus, the ratio of lithium ions in the outer electrode region and lithium ions in the inner electrode region is increasing with higher SOC.

This inhomogeneous lithium ion distribution is hypothesized to be the cause of the observed excess volume change [10]. During charging at high SOC, the higher concentration in the outer electrode region causes an enhanced volume expansion of the outer graphite particle. At the end of charge, no further lithium ions are inserted in the graphite particle whereas lithium ions are transferred from the outer electrode region to the inner electrode region. Over the rest phase, the lithium ion distribution steadily becomes more homogeneous and the excess volume change fades away.

This hypothesis also explains the strain signal and consequently the volume change observed during the CV charging phase. As described above, the monitored volume expansion 
reaches its maximum in the $\mathrm{CV}$ phase and subsequently starts decreasing. Initially, the charge current is almost similar to the charge current during the $\mathrm{CC}$ charge phase so that more lithium ions are inserted into the outer electrode region than transferred via diffusion to the inner region. However, the charge current is continuously decreasing over the CV phase. Thus, fewer lithium ions are provided to intercalate into the electrode over the CV phase and eventually more lithium ions diffuse into the inner electrode region. The steadily increasing level of homogeneity in lithium ion distribution gradually results in a fading of the excess volume change.

The diffusion process is known to be a strongly temperature dependent process. Various experiments at different temperatures in an environmental chamber are performed to analyze the temperature dependency of the strain relaxation. The settling time is set to $7 \mathrm{~h}$ to ensure thermal equilibrium. At first, standard cycles as described above are performed at $28^{\circ} \mathrm{C}, 32^{\circ} \mathrm{C}$ and $42^{\circ} \mathrm{C}$. The strain signal of the corresponding charge cycle is shown in Figure 4.

The FBG strain signals are zeroed for each temperature at the beginning of the charge cycle for comparison of the signals at the different temperatures. The strain signals are almost identical at low SOCs. All curves show the same features and exhibit the same wavelength shift up until SOC level of $65 \%$. Beyond SOC level of $65 \%$, however, the strain signals for the different temperature curves begin to diverge. The divergence starts in the range where the beginning of the excess volume change was identified in the previous experiment. The strain signal exhibits a steeper increase and consequently a higher volume change with decreasing temperature. Both observations are consistent with the assumption that the excess volume change depends on the lithium ion diffusion in the electrode materials. At higher temperatures, the faster lithium ion diffusion results in a more homogeneous lithium distribution in the electrode and consequently less strain is generated. 
As the lithium ion diffusion depends on the lithium gradient in the electrode particle the strain signal is examined for various charge currents at a fixed temperature. The inset in Figure 4 illustrates the wavelength shift for $\mathrm{C} / 2$ and $\mathrm{C} / 5$ constant current charge cycles as a function of SOC. Similar to the strain signals at various temperatures, both signals are identical for low SOCs and shift apart at higher SOC. In this case, the strain signal increases steeper with higher charge currents. This characteristic is consistent to the explanation given in Figure 5. The lithium intercalation is faster and the lithium ions have less time to diffuse for higher currents, which results in a more inhomogeneous lithium distribution.

The faster diffusion at higher temperatures should also result in a faster decay of the relaxation process in the subsequent no-load phase. Figure 6 illustrates the strain signals during the no-load phase at $28^{\circ} \mathrm{C}$ and $42^{\circ} \mathrm{C}$. The strain signal at $42^{\circ} \mathrm{C}$ converges faster to a constant value. The relaxation process observed in the extracted strain signal can be approximated by

$$
\Delta \lambda=\lambda_{0}+\lambda_{w h} e^{-\frac{\vec{t}}{\tau}}
$$

where $\Delta \lambda$ is the actual wavelength shift, $\lambda_{0}$ the residual wavelength shift after relaxation, $\lambda_{\text {rel }}$ the amount of relaxation and $\tau$ the time constant. The calculated time constants $\tau$ as a function of the inverse temperature for various temperatures are shown in Figure 7. The decreasing time constant with increasing temperature exhibits a faster decay of the relaxation process that is consistent with faster lithium ion diffusion.

Finally, the relation between strain relaxation and state of charge is investigated at various temperatures. For this purpose, the cell is stepwise charged with $\mathrm{C} / 2$ to various $\mathrm{SOC}$ levels similar to the experiment corresponding to Figure 3. The rest time is set to 2 hours for 
each SOC. The measurements were performed at $28^{\circ} \mathrm{C}, 32^{\circ} \mathrm{C}$ and $42^{\circ} \mathrm{C}$ and the settling time at each temperature is set to 7 hours.

Figure 8 illustrates the strain signals during no load after charging to various SOC. The strain signals at each SOC are labeled with a certain color, which brightens with increasing temperature. For example, for SOC100, black corresponds to $28^{\circ} \mathrm{C}$; dark grey corresponds to $32^{\circ} \mathrm{C}$, and light grey corresponds to $42^{\circ} \mathrm{C}$. The strain signals exhibit similar characteristics as the measurements at room temperature.

Again, the wavelength shifts indicate no distinctive relaxations at SOC levels of $10 \%$, $30 \%$ and $50 \%$, whereas at $80 \%$ and $100 \%$ clear relaxation is observable for all temperatures. The decay of the wavelength shift during the relaxation at $100 \%$ is more than twice in amplitude compared to the relaxation at $80 \%$. The absolute wavelength shifts of the strain signal at various temperatures line up for low SOCs whereas at higher SOCs the absolute wavelength shifts diverge for different temperatures.

For each SOC, the residual strain at the end of relaxation increases with decreasing temperature. For example, at SOC level of $100 \%$, the residual strains at the end of 2 hours of relaxation are $161 \mathrm{pm}, 113 \mathrm{pm}$ and $105 \mathrm{pm}$ for $28^{\circ} \mathrm{C}, 32^{\circ} \mathrm{C}$ and $42^{\circ} \mathrm{C}$ respectively. Moreover, the differences between the residual strains are higher at higher SOCs. Thus, there is an additional residual volume change beyond the excess volume change that is relaxing. As noted above, the additional volume change is dependent both on temperature and SOC. The additional volume change is increasing with decreasing temperature and increasing SOC.

A possible reason for this remaining volume change is the enhanced inhomogeneous lithium distribution in the graphite particles and an incomplete homogenization in the subsequent no-load phase. As described above, the lithium concentration gradient in the graphite particles 
causes diffusion and consequently to a more homogeneous lithium distribution. At higher temperatures, the lithium diffusion in the electrode materials is considerably faster. Thus, the lithium distribution is already more homogeneous during charging whereas at low temperatures, more lithium ions are concentrated in the outer region.

In general, lithium ions are intercalated between graphite layers in a particular order described as staging [14]. At high SOC and low temperatures, more lithium ions are concentrated in the outer region. Hence, lithium ions are intercalated in a different stage compared to the inner region of the graphite particle. The insertion of lithium ions into graphite is a thermodynamic process that requires energy to expand the van der Waals gap between two graphite layers. As the repulsive Coulombic interactions between the guest ions are less effective, few but highly occupied van der Waals gaps are energetically favored compared to randomly distributed guest ions [15]-[16]. Thus, further rearrangement via diffusion is initially energetically unfavorable so that excess volume change remains.

\section{Conclusions}

This study examined the excess volume change of a lithium ion pouch cell at the end of charge and the volume relaxation in the subsequent rest phase by monitoring the strain signal of externally attached FBG sensors. The excess volume change was only observed at high SOC. At the transition from charge to rest phase and immediate relaxation of the excess volume change was monitored. The excess volume change was explained by an inhomogeneous lithium ion distribution in the electrode active materials due to fast lithium intercalation in the outer region and slow diffusion in the electrode active materials. The influence of the inhomogeneous lithium distribution and the resulting excess volume change are only significant at high SOC levels. 
Thus, it is expected that the impact of the lithium diffusion is only distinguishable at high SOC as well.

The subsequent relaxation phenomena during no load were associated with lithium diffusion resulting in homogeneous lithium ion redistribution in the electrode particles. A correlation of the relaxation process and the lithium diffusion was indicated by an analysis of the time constants at various temperatures. These results are important to understand the impact of the excess volume change and the correlating relaxation with regard to the utility of the strain signal as input parameter for BMS. As mentioned earlier, the electrode volume change has been identified in the literature as a key reason for capacity fading. Thus, based on fundamental knowledge of the underlying electrochemical processes, charge methods and conditions can be optimized to avoid excess cell volume changes, and in turn, cell aging. This can help improve battery management and maximize pack performance and life.

\section{Acknowledgement}

The information, data, or work presented herein was funded in part by the Advanced Research Projects Agency-Energy (ARPA-E), U.S. Department of Energy, under Award Number DEAR0000274.

\section{References}

[1] T. Turrentine, Plug-in Hybrid Electric Vehicle Research Roadmap. UC Davis Plug-In Hybrid Electric Vehicle Research Center, June 2011. 
[2] K. A. Smith, C.-Y. Wang, Power and thermal characterization of a lithium-ion battery pack for hybrid-electric vehicles. J. Power Sources, 160, 662-673, 2006.

[3] N. A. Chaturvedi, R. Klein, J. Christensen, J. Ahmed, A. Kojic, Algorithms for advanced battery-management systems. Control Systems, IEEE, 30(3), 49-68, 2010.

[4] K. T. V. Grattan, T. Sun, Fiber optic sensor technology: an overview, Sensors and Actuators, Bd. 82, pp. 40-61, 2000..

[5] A. D. Kersey, A Review of Recent Developments in Fiber Optic Sensor Technology, Optical Fiber Technology, Bd. 2, pp. 291-317, 1996.

[6] L. W. Sommer, A. Raghavan, P. Kiesel, B. Saha, T. Staudt, A. Lochbaum, A. Ganguli, C.-J. Bae, M. Alamgir, Embedded Fiber Optic Sensing for Accurate State Estimation in Advanced Battery Management Systems. MRS Spring Meeting Symposium Q Proceedings, April 2014

[7] N. Zhang, H. Tang, Dissecting anode swelling in commercial lithium-ion batteries, Journal of Power Sources, Bd. 218, pp. 52-55, 2012..

[8] J. H. Lee, H. M. Lee, S. Ahn, Battery dimensional changes occurring during charge/discharge cycles — thin rectangular lithium ion and polymer cells, Journal of Power Sources, Bd. 1, 2119-121, pp. 833-837, 2003.

[9] M. Hahn, H. Buqa, P. W. Ruch, D. Goers, M. E. Spahr, J. Ufheil,P. Novak, R. Koetz, A Dilatometric Study of Lithium Intercalation into Powder-Type Graphite Electrodes, Electrochemical and Solid-State Letters, Bd. 11, Nr. 9, pp. A151-A154, 2008.

[10] X. Wang, Y. Sone, S. Kuwajima, In situ Investigation of the Volume Change in Li-ion Cell with Charging and Discharging, Journal of The Electrochemical Society, Bd. 151, Nr. 2, pp. 
A273-A280, 2004.

[11] X. Wang, Y. Sone, G. Segami, H. Naito, C. Yamada, K. Kibe, Understanding Volume Change in Lithium-Ion Cells during Charging and Discharging Using In Situ Measurements, Journal of The Electrochemical Society, Bd. 154, Nr. 1, pp. A14-A21, 2007.

[12] M. Fleckenstein, O. Bohlen, M. A. Roscher, B. Bäker, Current density and state of charge inhomogeneities in Li-ion battery cells with $\mathrm{LiFePO} 4$ as cathode material due to temperature gradients, Journal of Sources 196 (2011) 4769-4778

[13] Maire, P., Evans A., Kaiser H., Scheifele W., and Novák P., "Colorimetric determination of lithium content in electrodes of lithium-ion batteries," Journal of The Electrochemical Society 155, no. 11, A862-A865, 2008.

[14] V. A. Sethuraman, L. J. Hardwick, V. Srinivasan, and R. Kostecki, Surface Structural Disordering in Graphite upon Lithium Intercalation/Deintercalation, Journal of Power Sources, 195(11), 3655-3660, 2010.

[15] M. Winter, J. O. Besenhard, M. E. Spahr, P. Novak, Insertion Electrode Materials for Rechargeable Lithium Batteries, Advanced Materials, Bd. 10, Nr. 10, pp. 725-763, 1998.

[16] R. A. Huggins, Lithium-Carbon Alloys, Advanced Batteries, Springer, 2008.

\section{Figure Captions}

1. Schematic of the experimental setup for monitoring strain and temperature of Li-Ion pouch cells by Fiber Bragg Grating sensors. Bonded FBG sensor is attached with an 
adhesive to monitor strain and temperature. Reference FBG sensor is loosely attached with heat conducting paste.

2. Bragg wavelength shift of the bonded (black) and loosely attached (red) FBG sensor as well as the temperature compensated strain signal (blue) for standard charge and discharge. The corresponding voltage and current data are illustrated in the upper plot. A wavelength shift of $1 \mathrm{pm}$ corresponds to a temperature change of $\sim 0.1 \mathrm{C}$ or an elongation of $\sim 1 \mu \varepsilon$.

3. (a) Wavelength shift of bonded FBG and reference FBG sensor as well as the extracted strain signal due to the electrode expansion caused by $\mathrm{Li}$ intercalation for a stepwise $\mathrm{C} / 2$ charge to SOC levels of 10\%, 30\%, 50\%, 80\%, and 100\% (here "SOCx" implies SOC level of $\mathrm{x} \%$, while "C" indicates charge phase); (b) Zoomed-in strain signal during rest (no load) period after charging to various SOC. At SOC levels of 10\%, 30\% and 50\% almost no relaxation is observable whereas a distinctive relaxation behavior is observable at $80 \%$ and $100 \%$.

4. Strain signal for standard charge cycle at various temperatures. The inset figure shows the strain signal as a function of SOC for two different charge rates $(\mathrm{C} / 2$ and $\mathrm{C} / 5)$. The strain signals for different temperatures and different C-rates line up for low SOC (up to $\sim 65 \%$ ). At high SOC, the strain signals increase steeper with decreasing temperature and higher C-rates. 
5. Schematic illustration of the hypothesis to explain the observed excess volume change, as initially proposed by Wang et al. [10]. The excess volume change takes place at high SOC due to an inhomogeneous distribution of lithium ions in the graphite particle. The inhomogeneous distribution is caused by fast lithium ion intercalation and slow diffusion in that particle. During no load, lithium ions are transferred to the inner region of that particle and the excess volume relaxes.

6. Strain signal relaxation during no load after charging at $28^{\circ} \mathrm{C}$ (upper plot) and $42^{\circ} \mathrm{C}$ (lower plot).

7. Time constant $\tau$ of the relaxation process at SOC level of $100 \%$ as a function of temperature. The time constant increases with decreasing temperature which equals a slower strain decay.

8. Strain signals during no load after charging to various $\mathrm{SOC}$ at $28^{\circ} \mathrm{C}, 32^{\circ} \mathrm{C}$ and $42^{\circ} \mathrm{C}$. The strain signals at each SOC are labeled with a certain color which brightens with increasing temperature. At SOC level of $10 \%, 30 \%$ and $50 \%$ almost no relaxation is distinguishable whereas distinctive relaxations are monitored at $80 \%$ and $100 \%$. The absolute wavelength shifts of the strain signal at various temperatures line up for low SOCs whereas at higher SOCs the absolute wavelength shifts diverge for different temperatures. The strain signals exhibit an additional wavelength shift with decreasing temperature. 


\section{Figures}

Figure 1:

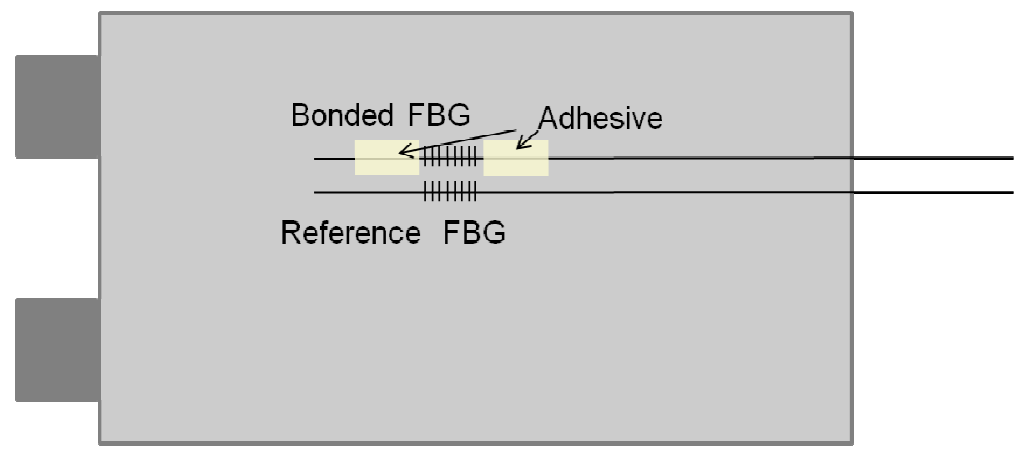

Figure 2:

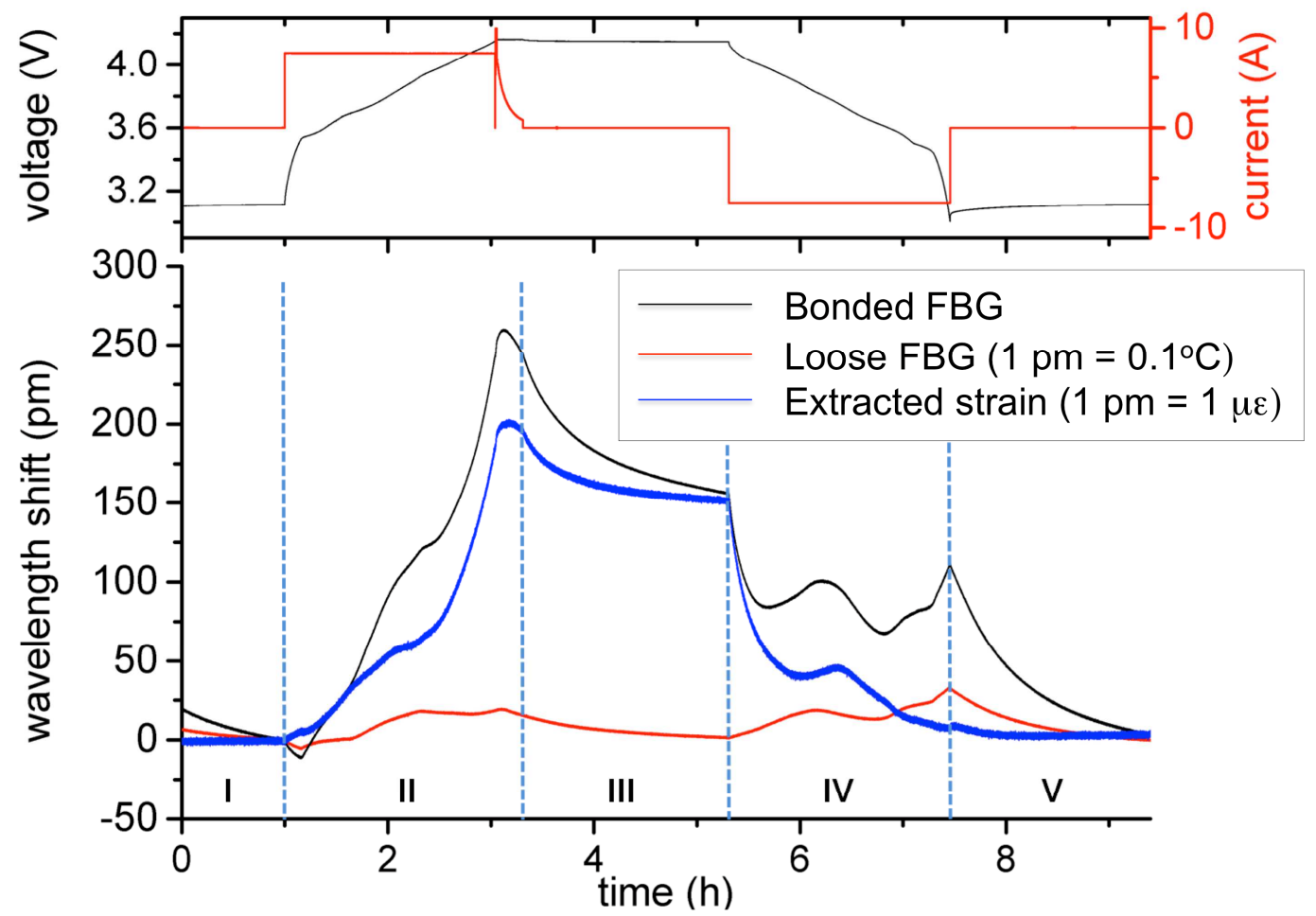


Figure 3:

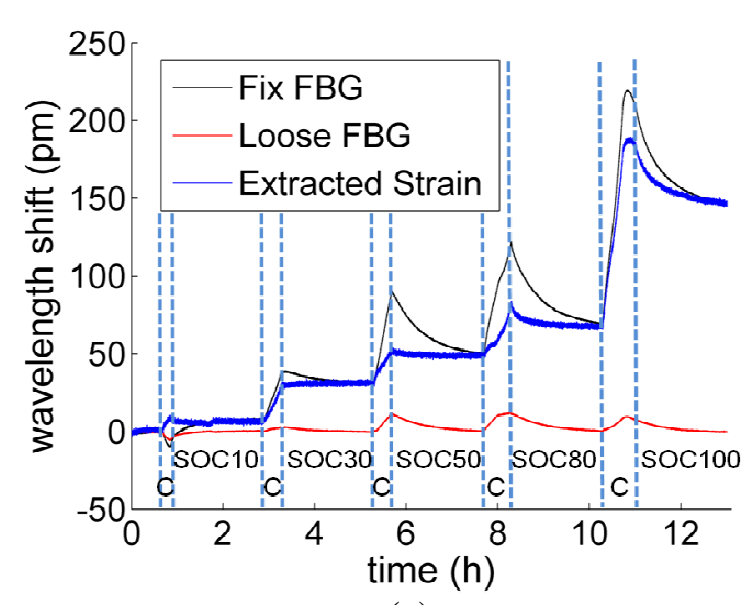

(a)

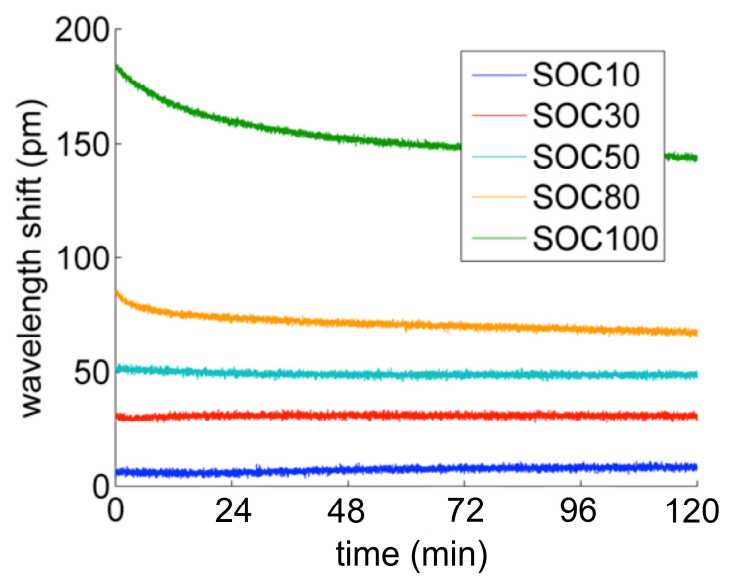

(b)

Figure 4:

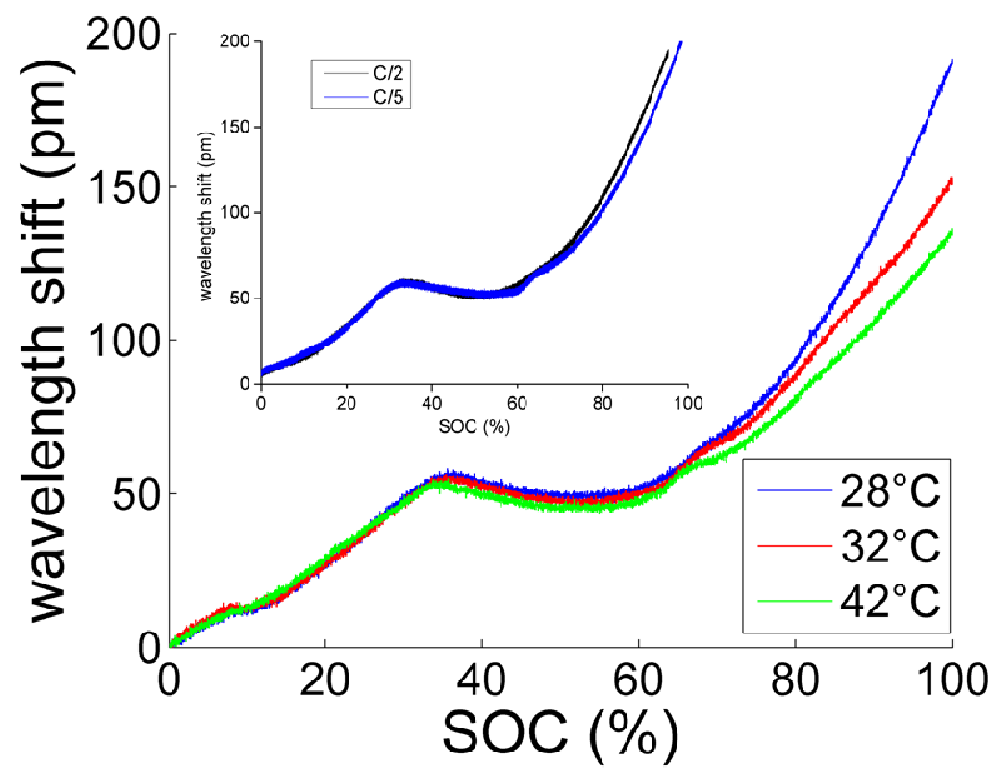


Figure 5:

a)

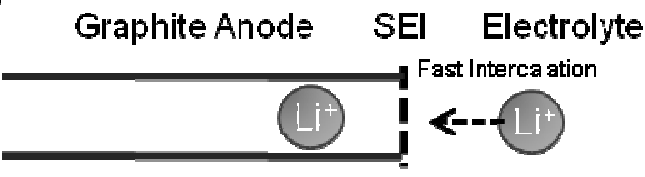

b) Graphite Anode SEI Electrolyte

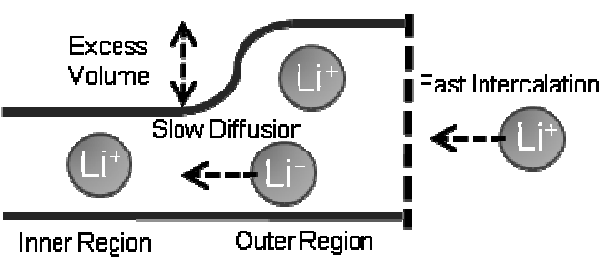

c)

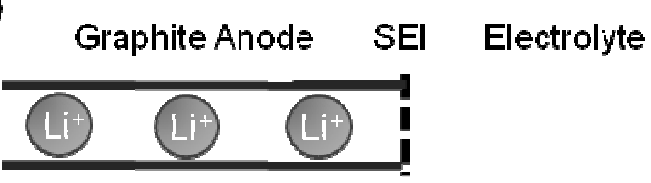

Figure 6:

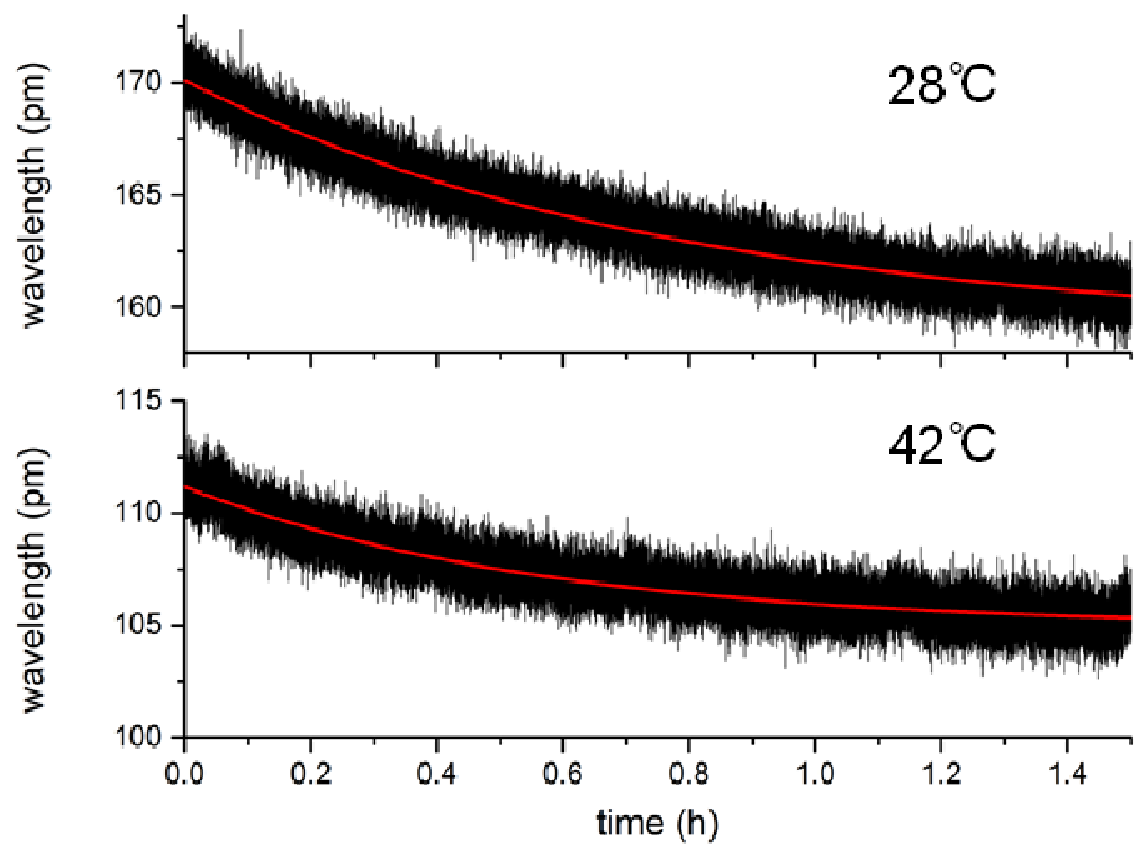


Figure 7:

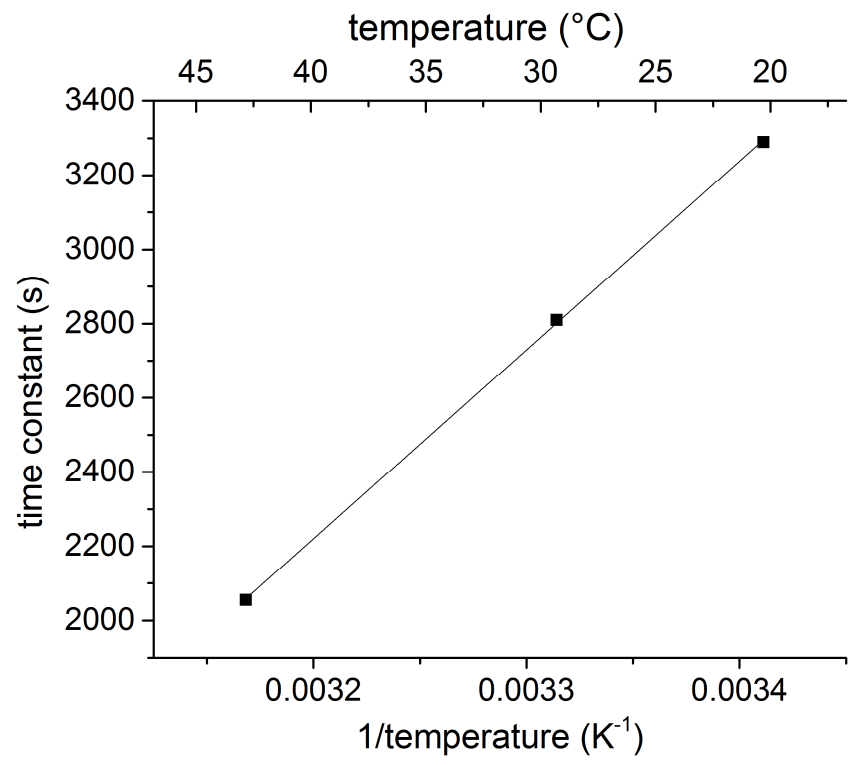

Figure 8:

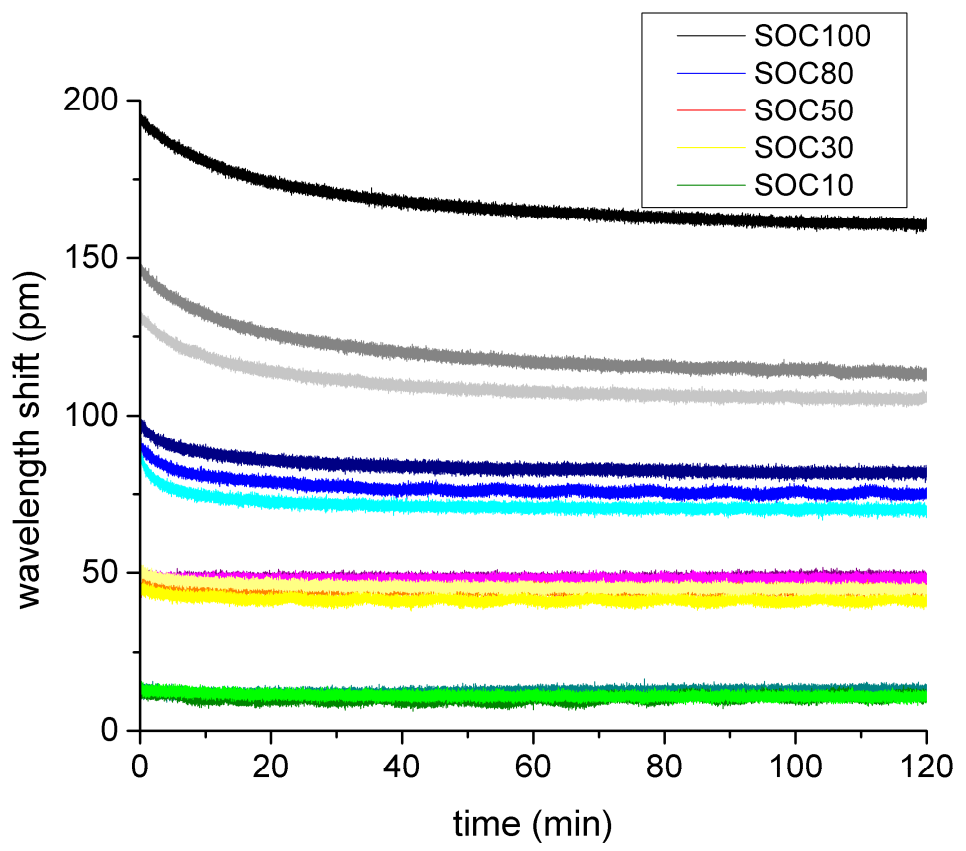

